\title{
Strates
}

STRATES Matériaux pour la recherche en sciences sociales

9 | 1997

Crises et mutations des territoires

\section{Les aveugles et l'éléphant : l'explication de la gentrification}

\section{Chris Hamnett}

\section{(2) OpenEdition}

1 Journals

Édition électronique

URL : http://journals.openedition.org/strates/611

DOI : $10.4000 /$ strates.611

ISSN : $1777-5442$

Éditeur

Laboratoire Ladyss

Édition imprimée

Date de publication : 30 septembre 1997

ISSN : 0768-8067

Référence électronique

Chris Hamnett, "Les aveugles et l'éléphant : l'explication de la gentrification », Strates [En ligne], 9 |

1997, mis en ligne le 19 octobre 2005, consulté le 08 septembre 2020. URL : http://

journals.openedition.org/strates/611; DOI : https://doi.org/10.4000/strates.611

Ce document a été généré automatiquement le 8 septembre 2020

Tous droits réservés 


\title{
Les aveugles et l'éléphant: l'explication de la gentrification
}

\author{
Chris Hamnett
}

Introduction

1 Au cours des dix dernières années, le phénomène de gentrification et le débat sur sa signification, ses processus, son explication et ses effets ont occupé une place particulièrement importante dans les revues (Hamnett, 1984 ; Smith and Williams, 1986 pour des bibliographies récentes). Dans les seules Annals of the American Association of Geographers ont paru des articles de Ley $(1980,1986,1987)$, de Schaffer et Smith (1986), de Smith (1987b) et de Badcock (1989).

2 La gentrification est désormais identifiée dans un grand nombre de villes d'Amérique du Nord, d'Europe et d'Australie. Néanmoins, en dépit de son expansion au cours des années 1970 et 1980, il s'agit d'un phénomène d'une ampleur encore limitée qui reste très concentré géographiquement, contrairement aux phénomènes de suburbanisation de l'après-guerre et du déclin des centres-villes. B. Berry (1985) les définit (par défaut) comme îlots de rénovation dans des mers de déclin. Aussi est-il important de se demander pourquoi tant d'attention a été accordée à ce thème. Cinq raisons au moins peuvent être identifiées ; elles seront présentées par ordre d'importance croissant.

3 Tout d'abord, et d'un point de vue en quelque sorte utilitaire, la gentrification a offert un thème de recherches commode à une nouvelle génération de géographes et de sociologues urbains en quête de sujets de recherche inédits et potentiellement intéressants concernant la ville. Ceci explique le grand nombre d'études de cas limitées à une dimension locale qui sont restées sans suite.

4 La deuxième explication, plus convaincante, est que la gentrification a posé un défi majeur aux théories classiques de la localisation résidentielle et des structures sociales urbaines (Hamnett, 1984). Le changement des quartiers a été conçu par H. Hoyt et par E. Burgess comme un processus irréversible dans lequel «les riches reviennent rarement sur leurs pas, pour retrouver les logements obsolètes qu'ils avaient abandonnés » (Hoyt, 1939, p.118). La gentrification réfute le postulat majeur selon lequel le filtrage est un processus unidirectionnel descendant, par lequel les groupes à faibles revenus 
s'installent dans un habitat en cours de détérioration. Ce modèle remet également en cause l'hypothèse sur laquelle repose la théorie structurelle du marché foncier urbain d'Alonso, selon laquelle on privilégie l'espace et les faibles densités plutôt que l'accessibilité aux centres-villes. Enfin, la gentrification remet en question les précédentes "théories des étapes" ou modèles d'évolution du changement urbain résidentiel, qui font de la suburbanisation des couches moyennes le stade final du processus de passage de la ville préindustrielle à la ville industrielle. Ley, en 1981, a commenté ceci comme le résultat du "processus de revitalisation de la décennie précédente, au cours de laquelle, dans certains quartiers des centres d'agglomération postindustriels, les quartiers de logements ouvriers étaient transformés en quartiers privilégiés rappelant l'anneau résidentiel central du modèle de ville préindustrielle proposé par Sjoberg. Si les tendances actuelles se poursuivent, la géographie sociale de la ville industrielle du XIXe siècle peut même apparaître aux chercheurs en urbanisme du futur comme une étape transitoire vers un schéma historiquement plus stable de ségrégation de haut statut adjacent au cour des agglomérations. " (Ley, 1981, p. 145).

5 La troisième raison de l'émergence de la gentrification comme thème de recherche majeur tient à l'apparition d'une politique et de débats politiques concernant les déplacements de population que ce processus implique. Alors que certains ont perçu la gentrification comme un salut inespéré pour les centres-villes, marquant un arrêt de l'abandon résidentiel après des décennies de fuite des classes moyennes blanches et permettant une augmentation de la base fiscale (Sumka, 1979; Sternlieb et Hughes, 1983), d'autres la considèrent en revanche comme une menace sur les zones résidentielles ouvrières des centres-villes (Ley, 1981 ; Hartman, 1979 ; Marcuse, 1986 ; Legates et Hartman, 1986) et un prélude à la reconversion de parties entières des centres-villes en parcs de jeux pour la bourgeoisie (Schaeffer et Smith, 1986).

Une quatrième explication, liée à la précédente, est que la gentrification constitue l'un des principaux mouvements dans la restructuration métropolitaine contemporaine. De la même manière que la suburbanisation et le déclin des centres-villes constituaient les processus caractéristiques de la restructuration urbaine dans les années 1950 et 1960, la gentrification est censée représenter l'un de ces processus dans les années 1970 et 1980. En ralentissant ou en inversant la diminution des couches moyennes et la détérioration des logements dans les centres-villes, la gentrification va à contre-courant des tendances précédentes. Dans cette perspective, la gentrification, à l'instar de la suburbanisation dans les décennies précédentes, met en lumière l'importance des mouvements de capitaux entre les secteurs économiques et les différents quartiers de la ville (Smith, 1979; Harvey, 1978; Badcock, 1989; King, 1989a, 1989b, 1989c). Cet argument est développé par Smith et Williams (1986) qui suggèrent, entre autres, que la gentrification doit être considérée comme l'un des changements de la division internationale du travail, avec l'émergence des villes globales, qui doivent être considérées, par le contrôle et les fonctions de commandement qu'elles exercent, comme parties d'une nouvelle hiérarchie urbaine dominée par des flux de capitaux financiers. De ces processus découle la restructuration simultanée de la hiérarchie urbaine et de l'espace intra-urbain. Schaeffer et Smith (1986) rejettent ainsi la position des minimalistes tels que B. Berry qui ne veulent voir dans la gentrification qu'un processus à grande échelle. Ils considèrent que "nous ne sommes pas les témoins d'une curieuse anomalie mais d'une restructuration profonde de l'espace urbain » (Schaffer et Smith, 1986, p. 362). 
7 La cinquième raison, discutable, est aussi la plus importante explication de la place prise par la gentrification dans les travaux de géographie urbaine contemporaine : la gentrification représente l'un des champs de bataille théoriques et idéologiques majeurs en géographie urbaine, donc dans la géographie dans son ensemble, entre les humanistes libéraux qui mettent en avant le rôle-clé du choix, de la culture, de la consommation et de la demande de consommation, et les marxistes structuralistes qui insistent au contraire sur le rôle du capital, des classes, de la production et de l'offre. La gentrification est l'un des lieux principaux de ce conflit entre les tenants de la culture, de la préférence et de l'action individuelle, et les défenseurs des impératifs du capital et du profit. En effet, deux des principaux protagonistes, D. Ley et N. Smith, se sont par ailleurs étroitement engagés dans des débats beaucoup plus larges sur l'épistémologie et l'explication en géographie humaine (Duncan et Ley, 1982 ; Smith, 1982, 1987c).

Dans la mesure où cette interprétation est correcte, la gentrification constitue une frontière (Smith, 1986), non seulement physique, économique, sociale et culturelle, mais aussi théorique, idéologique et politique. Elle comprend une zone-frontière disputée entre des théories et des schémas explicatifs radicalement différents. Et c'est probablement cet aspect de la gentrification qui, plus que tout autre, a placé le débat sur le devant de la scène en géographie urbaine depuis plus d'une décennie. Le débat sur la gentrification se joue autour des enjeux théoriques et idéologiques importants. Il n'est pas surprenant que ce débat ait été si vif entre tenants de la production et de la profitabilité, et tenants de la consommation et du choix. Comme Schaeffer et Smith l'expriment sans ambages en 1986 : «Le débat sur les causes de la gentrification en est arrivé à se centrer sur les explications fondées sur la production ou sur la consommation. Chacune de ces positions implique un engagement théorique plus conséquent sur la façon dont l'espace urbain est continuellement modelé et remodelé." (Schaffer et Smith, 1986, p. 350).

Et dans sa critique favorable aux approches marxistes de la gentrification, Rose note que : "Les travaux marxistes sur la gentrification ont insisté sur le fait que le point de départ "acceptable" à partir duquel théoriser ce processus est celui de la production de logements gentrifiés. J'utilise le terme "acceptable" pour attirer l'attention sur le fait que cette façon d'insister sur un unique point de départ analytique dans "la sphère de production" a des fondements politiques. Dans cette sphère, les activités sont... le principal moteur des changements dans la société capitaliste... Dans une large mesure, les approches marxistes de la gentrification ont déterminé d'avance leurs objectifs, par opposition aux approches positivistes... Contrairement à ces dernières, il faut noter que, dans l'approche marxiste, la gentrification est considérée comme un processus non réductible aux seuls comportements des individus. " (Rose, 1984, pp. 49-50).

10 C'est cet aspect de la gentrification comme champ de bataille intellectuel entre des perspectives théoriques rivales et irréductiblement opposées que j'ai l'intention de privilégier dans ce texte. Bien que de nombreuses explications alternatives aient été proposées (Hamnett, 1984 ; Ley, 1986 ; Smith, 1986), notamment celles concernant des changements dans la démographie, les styles de vie, les équipements collectifs, les dynamiques des marchés fonciers et du logement ou celles de l'activité économique et des structures d'emploi, ces explications peuvent être réparties en deux grandes séries concurrentes. La première, surtout associée aux travaux de Smith, met l'accent sur la production de l'espace urbain, le fonctionnement des marchés foncier et immobilier, sur le rôle du capital et des acteurs collectifs tels que les promoteurs et les institutions de crédit foncier et de prêts hypothécaires pour l'offre de propriétés à gentrifier. La 
seconde, désignée par Smith sous le terme d'argument du côté de la consommation, met l'accent sur la production des gentrifieurs et sur leurs orientations en matière de culture, de consommation et de reproduction (Ley, 1980 ; Mullins, 1982 ; Moore, 1982 ; Rose, 1984 ; Williams, 1984 ; Beauregard, 1986).

Ma thèse est ici que ces différentes perspectives théoriques sur la gentrification constituent des abstractions partielles de ce phénomène, qui mettent l'accent sur des aspects différents et cependant tous cruciaux. Comme dans la fable d'Ésope sur les aveugles et l'éléphant, chacune de ces théories ne perçoit qu'une partie de l'éléphant de la gentrification. Les deux perspectives théoriques sont plus complémentaires que concurrentes. C'est un point dont on a peu à peu apprécié l'importance et les tendances exclusives premières ont été abandonnées. Une théorie intégrée de la gentrification (Hamnett, 1984; Beauregard, 1986) s'est progressivement constituée lorsque les chercheurs ont réalisé combien production et consommation étaient toutes deux cruciales pour une explication exhaustive.

Dans l'exposé de cette thèse, nous n'accorderons qu'une attention limitée aux débats sur le rôle de l'État dans la gentrification et à la dimension du genre (gender) dans ce processus (Rose, 1984 et 1989). Bien entendu ces enjeux sont importants, mais ils restent secondaires en comparaison de la difficulté majeure posée par le rapport entre la production et la consommation. Certes Cybriwsky et al. (1986) comme Smith (1989) considèrent que le rôle de l'État est important pour la compréhension des processus de gentrification dans certains quartiers. Cependant un grand débat s'est ouvert concernant l'importance relative à accorder aux acteurs individuels, à leurs motivations et au rôle structurel de l'État. Aussi le débat sur le rôle de l'État dans la gentrification reflète et incarne le débat plus large sur la gentrification entre tenants de la structure et tenants de l'action (Gregory, 1981).

Ce texte comprend neuf parties. La première propose une définition de la gentrification et en expose les critères explicatifs. Les deuxième et troisième parties présentent et critiquent l'approche proposée par D. Ley. Les quatrième et cinquième parties évaluent la thèse du différentiel de loyer de Smith. La sixième section met en valeur l'importance de la production des gentrifieurs et leurs localisations préférentielles. La septième section examine les reformulations de Smith et sa tentative d'intégrer la consommation dans son schéma théorique, et la huitième partie insiste sur les éléments d'une approche intégrée. Enfin la dernière section résume et conclut le débat.

Gentrification : une définition et des critères d'explication

Avant de présenter et analyser les thèses concurrentes, nous devons d'abord définir la gentrification et établir les critères pour une explication complète, par rapport à laquelle les différentes théories pourront être évaluées. Hamnett (1984, p. 284) définit ainsi la gentrification "(comme) un phénomène à la fois physique, économique, social et culturel. La gentrification implique en général l'invasion de quartiers auparavant ouvriers ou d'immeubles collectifs en dégradation par des groupes de classes moyennes ou aisées et le remplacement ou le déplacement de beaucoup des occupants originaux de ces quartiers. Cela implique la rénovation ou la réhabilitation physique de ce qui était auparavant un stock de logements très dégradés et son amélioration pour convenir aux besoins des nouveaux occupants. Au cours de ce processus, le prix des logements situés dans les quartiers concernés, réhabilités ou non, augmente fortement. Un tel processus de transition des quartiers implique en règle générale un certain degré de transformation des statuts d'occupation, de la location à la propriété occupante.» 
Smith (1987b, p. 463) estime que «Le point le plus important est que la gentrification implique non seulement un changement social mais aussi un changement physique du stock de logements, à l'échelle des quartiers, enfin un changement économique sur les marchés foncier et immobilier. C'est cette combinaison de changements sociaux, physiques et économiques qui distingue la gentrification comme processus ou ensemble de processus spécifiques. "

Selon ces perspectives, il est clair que la gentrification implique à la fois un changement dans la composition sociale des résidents d'un quartier, et un changement dans la nature du parc de logements (en particulier dans le statut d'occupation, les prix, les conditions, etc.). Aussi une explication adéquate de la gentrification doit-elle concerner ces deux aspects du processus, c'est-à-dire les logements et les résidents. Afin de passer de ces définitions à la spécification des critères d'explication de la gentrification, je propose que toute explication d'ensemble se doit de rendre compte des quatre aspects principaux de ce processus :

1. Pourquoi la gentrification est-elle particulièrement concentrée dans un petit nombre de grandes villes comme Paris, Londres, New York, San Francisco, Toronto, Sydney, Melbourne, et pourquoi est-elle plus limitée dans des villes industrielles plus anciennes?

8 2. Pourquoi la gentrification apparaît-elle dans certains quartiers et dans certains stocks de logements et non dans d'autres, et quelles sont les caractéristiques des quartiers concernés?

3. La théorie doit préciser et expliquer quels groupes sociaux deviennent des gentrifieurs.

4. La théorie doit expliquer les étapes de la gentrification.

En d'autres termes, une explication complète de la gentrification doit répondre aux questions suivantes : quels quartiers, où, quand et pourquoi ?

2 Je me propose ici de montrer que chacun des grands schémas explicatifs traite ou répond à l'une ou l'autre de ces questions mais non à toutes. En effet, on peut considérer que chacun de ces schémas, bien qu'il ait un pouvoir explicatif considérable, est cependant incapable de répondre seul à toutes ces questions, à cause de son point focal et de son ampleur. C'est pourquoi ces schémas ne constituent que des explications partielles, dont la validité est limitée. Comme les travaux de Smith (1979) sont en partie une réponse à des travaux de D. Ley (1978) et d'autres chercheurs, nous considérerons d'abord les travaux de D. Ley.

Culture et consommation dans la ville postindustrielle

23 En 1980, D. Ley publiait Liberal ideology and the post-industrial city. Dans cet ouvrage, il introduit ce qui peut être considéré rétrospectivement comme un schéma théorique-clé sur les origines et les causes de la gentrification, bien que le thème principal traite de la montée du Electors Action Movement à Vancouver, et de la politique de restriction de l'activité immobilière et de la construction d'autoroutes que ce mouvement a mené, pour créer une ville agréable à vivre.

24 Dans ce texte, qui apparut plus tard comme la cible fétiche des analystes marxistes, D. Ley estimait qu'une nouvelle idéologie du développement urbain était en train de se constituer. La stratégie urbaine semblait se concentrer désormais sur la croissance plutôt que sur la qualité de la vie; on reconnaissait moins le nouveau libéralisme à ses rythmes de production qu'à ses styles de consommation (Ley, 1980, p. 239). 

un vaste ensemble de processus de changements dans la société» (Ley, 1980, p. 240). Pour les identifier, D. Ley a repris les travaux de D. Bell sur la société postindustrielle et ceux d'Habermas sur le capitalisme avancé. Il reconnaissait qu'il s'agissait là de compagnons théoriques improbables, mais estimait qu'il y avait «une complémentarité plus profonde dans leurs positions. Ils conçoivent tous deux une transition décisive entre le XIX et la fin du XX siècle, entre la période industrielle du capitalisme précoce, et la période postindustrielle du capitalisme tardif.» (p. 240). l'économie, la politique et la culture. Comme nous le verrons plus loin, l'ordre de ces propositions est important. D'abord, au niveau de l'économie, le rôle déclinant du travail peu qualifié dans le procès de production et l'importance croissante de la technologie dans les usines, les bureaux et les administrations constituent une rupture majeure avec le $\mathrm{XIX}^{\mathrm{e}}$ siècle. Cette coupure a été associée à une transformation majeure de la force de travail, caractérisée par le déclin des cols bleus et la croissance des cols blancs, en particulier dans les strates les plus qualifiées - cadres moyens et supérieurs des fonctions d'administration-gestion et des fonctions techniques. Ley liait ceci au passage d'une société productrice de biens à une société productrice de services, et au déclin de l'industrie manufacturière et à la croissance du travail de bureau.

La deuxième proposition était que la société postindustrielle se distingue de la société industrielle par le rôle actif du gouvernement. En conséquence, Ley plaidait que la classe politique et non plus seulement les lois du marché étaient à l'origine de la prise de décision et de l'allocation des ressources. La politisation de groupes d'intérêt divers met en péril la main mise du lobby du milieu des affaires sur la prise de décision politique (Ley, 1980, p. 24). Troisièmement, Ley avançait qu'au niveau socioculturel, on pouvait remarquer que le rôle de l'individualité s'était à nouveau renforcé et que s'était développée une philosophie plus sensuelle, plus esthétique, parmi les représentants de plus en plus nombreux de la classe de service nord-américaine résidant sur la côte ouest. Il concluait que "nous pouvons tirer de ce schéma l'existence d'un groupe d'acteurs théoriquement significatifs... (qui) forment un contrepoint théorique aux notions, datant du XIX siècle, de capital et de travail... une classe en émergence... Avec une base économique sûre, ils représentent les contrepoints contemporains de la classe de loisir de Veblen, arborant les canons $d u$ bon goût, fondés sur l'esthétique. Leur style de vie est orienté vers la consommation et le statut, dans une quête d'auto-actualisation. » (Ley, 1980, pp. 242-243).

La référence de Ley à une " classe en émergence » est importante. Il note que, parce que la thèse postindustrielle a été développée par des sociologues, elle n'a pas reçu de précisions sur le plan géographique. Or il montrait que ces «traits ne sont pas distribués uniformément; il y a une géographie de la société postindustrielle... qui doit mieux correspondre aux caractéristiques de San Francisco ou de Londres, qu'à celles de Glasgow ou de Cleveland" (Ley, 1980, pp. 242-243). C'est un point central qui a des conséquences importantes sur la détermination des lieux où se produit la gentrification. Ley tenta d'appliquer cette thèse à Vancouver, recherchant quels ont été les changements apparus dans les structures industrielles, démographiques et économiques, comme dans les styles de vie et dans le marché du logement au centre de l'agglomération. Ley n'utilisait pas explicitement ce terme dans ce texte, mais en 1981 il établit une relation claire entre la croissance des secteurs tertiaire et quaternaire, la croissance des emplois de cadres 
moyens et supérieurs, les transformations de la demande de logements dans Vancouver, et la gentrification.

Ainsi qu'il le pose : «il est possible de suivre la transmission d'ajustements à grande échelle dans l'économie jusqu'au schéma de création d'emplois à Vancouver, avec des tendances favorisant la croissance des emplois de cols blancs dans le Central Business District. Ces facteurs contextuels sont sous-jacents aux changements démographiques dans l'aire métropolitaine et aux pressions sur la demande de logements qui les ont accompagnées. » (Ley, 1985, p. 128).

Mais ces pressions sur la demande de logements sont géographiquement circonscrites. S'interrogeant sur le nombre croissant de petits ménages jeunes et aisés et sur leur impact sur le marché du logement dans le centre de l'agglomération, Ley démontre que les facteurs culturels sont importants: "les quartiers eux-mêmes offrent une diversité de styles de vie, une diversité ethnique et architecturale, autant d'attributs valorisés par des immigrants des couches moyennes dans le centre de l'agglomération... Ces desiderata de la culture de consommation ne devraient pas être sous-estimés dans l'interprétation de la revitalisation des centres » (Ley, 1981, p. 128).

31 Ley s'attarde moins sur la structure et le fonctionnement des marchés foncier et immobilier, sur l'offre et la production de propriétés gentrifiables et sur les quartiers dans lesquels celles-ci sont situées. Lorsqu'il le fait, il met l'accent sur la demande. Ley notait le rôle de l'industrie immobilière, mais il lui accordait un rôle secondaire dans le processus de gentrification. En se référant à la revitalisation du centre de Kitsilano, il indique que "l'activité de l'industrie immobilière contribue sans aucun doute à l'instabilité du marché local du logement en accélérant le processus de transition et en alimentant la spirale inflationniste des valeurs foncières par la spéculation, et en augmentant les espoirs des propriétaires de vendre leurs maisons à des prix inespérés » (Ley, 1981, p. 138).

La priorité causale est claire. Ley considère que l'activité immobilière est stimulée par le pouvoir d'achat de la croissance des cols blancs, résultant des changements dans les structures économiques et d'emplois. Il reprend cette perspective dans un texte plus récent (1986) et pose que "la croissance de l'emploi dans le complexe des sièges sociaux situés dans les centres directionnels, les services aux entreprises, et indirectement dans les administrations publiques des centres nodaux contribue à la production de cadres moyens et supérieurs et à celle d'employés du secteur quaternaire travaillant dans les centres d'affaires. Ces actifs constituent ainsi la base de la demande de réinvestissement des logements des centresvilles. Cette population, lorsqu'elle exprime ses préférences pour les équipements centraux, sur les plans politique et économique, restructure l'environnement bâti et accélère le processus de gentrification.» (Ley, 1986, p. 532).

Une évaluation de la théorie de Ley sur l'urbanisme postindustriel

Dans la thèse de Ley, beaucoup d'arguments pourraient être remis en question par des marxistes, en particulier l'importance politique accordée à une nouvelle élite de fabricants de goûts et de faiseurs d'opinions, sur l'importance de la culture et de la consommation, sur l'acceptation de l'idée de postindustrialisme (Walker et Greenberg, 1982) et sur la relégation apparente de la production de l'environnement bâti et des notions, datant du XIX ${ }^{e}$ siècle, de capital et de travail, à un rôle secondaire dans les affaires urbaines.

34 Ley ne prône cependant pas une théorie du développement ou du changement urbain déterminé par la consommation, ou une théorie de la gentrification fondée sur les préférences des consommateurs, comme certains de ses détracteurs l'ont suggéré. Il ne fonde pas non plus exclusivement son travail sur le concept de ville postindustrielle, tel 
qu'il avait été élaboré par D. Bell. Au contraire l'importance accordée à la culture et à la consommation dans la ville postindustrielle est fortement enracinée dans les structures de production, dans les changements de la division du travail et dans l'émergence et la croissance d'une classe de services fortement concentrée géographiquement.

Ley montre que cette classe joue un rôle-clé dans la politique et dans la culture, il la considère aussi comme une conséquence des changements dans la division du travail et dans la nature spatialement inégale de ces changements. Ainsi lie-t-il les changements dans l'organisation de la production et l'économie, la politique et la culture, dans une approche de la gentrification et du changement urbain fondée sur la production des gentrifieurs, leurs caractéristiques et leurs exigences culturelles. Sans ces précautions, on aurait pu reprocher à $\mathrm{D}$. Ley de proposer une théorie de la gentrification non matérialiste, basée sur la consommation, comme ses détracteurs le suggéraient. A mon avis, ces critiques ont mal interprété l'accent mis sur la culture et la consommation, le considérant comme une approche étroite, fondée sur la préférence et la demande. En réalité, cette théorie est fondée sur les changements dans la division sociale et spatiale $\mathrm{du}$ travail et dans l'offre de gentrifieurs potentiels. Ces changements soutiennent le développement d'une nouvelle culture, et les demandes de logement et l'attente politique en découlent.

36 L'intérêt des premiers travaux de Ley tient à l'importance accordée aux changements dans les divisions spatiales et sociales du travail et dans la concentration d'une élite de cadres moyens et supérieurs dans un nombre limité de villes postindustrielles dominées par les services. D. Ley accorde une grande place au rôle des changements dans la culture et les modes de consommation et aux contraintes ou aux demandes résidentielles de la nouvelle élite, mais il les replace dans un contexte caractérisé par des changements dans la nature et dans les structures de l'organisation économique. La thèse de Ley est plus forte dans l'explication qu'elle offre du type de ville dans laquelle se produit la gentrification et dans les caractéristiques des gentrifieurs. Elle traite aussi implicitement de la temporalité de la gentrification à travers l'analyse de la croissance de l'économie de services dans les années 1970 et 1980 . Sa thèse est plus fragile dans l'explication des zones dans lesquelles la gentrification se produit puisque D. Ley les considère largement comme un produit de la demande pour des localisations en centres-villes et pour les équipements collectifs et culturels que ceux-ci offrent aux gentrifieurs. L'offre de logements potentiellement gentrifiables est supposée suivre la demande et le pouvoir d'achat des gentrifieurs qui leur permet de surpasser d'autres utilisateurs. Mais l'accent mis par Ley sur le pouvoir d'achat de la nouvelle élite suggère qu'il considère le pouvoir d'éliminer d'autres usagers comme un déterminant majeur du paysage urbain - peut-être aussi important que la culture de consommation de cette nouvelle élite.

L'analyse du côté de l'offre : gentrification et différentiel de loyer

La théorie proposée par D. Ley pour l'explication de la gentrification est fondée sur la production de gentrifieurs et sur ses conditions culturelles et de consommation comme éléments-clés. L'offre de propriétés gentrifiables et le fonctionnement des marchés foncier et immobilier ne jouent, dans cette perspective, qu'un rôle secondaire. Smith (1979) a complètement inversé le schéma explicatif, en montrant que l'argument des préférences des consommateurs était considéré comme acquis et contradictoire. Dans sa perspective, les actions des producteurs comme des consommateurs doivent être prises en compte pour expliquer le processus de gentrification. « La théorie selon laquelle 
il est possible d'expliquer la gentrification en fonction des seules actions des gentrifieurs, tout en ignorant le rôle des agents immobiliers, des promoteurs, des propriétaires, des prêteurs et des agences gouvernementales, des locataires, est terriblement limitée. Une théorie plus large de la gentrification doit prendre en compte le rôle des producteurs aussi bien que celui des consommateurs. » (Smith, 1979, p. 540.)

Smith a tout à fait raison sur ce point que Ley avait largement omis de prendre en compte. Mais Smith va plus loin, prêchant pour la domination du producteur : «Il apparaît que les besoins de la production - en particulier le besoin de dégager du profit - sont un facteur plus décisif de la gentrification que la préférence des consommateurs. Je ne considère pas naïvement que la consommation est la conséquence directe de la production ou que les préférences des consommateurs sont passivement déterminées par la production. Telle serait une théorie de la souveraineté des producteurs, presqu'aussi unilatérale que sa contrepartie néoclassique. En fait, la relation entre production et consommation est symbiotique, mais c'est une symbiose dans laquelle la production domine. Bien qu'elles soient secondaires dans l'origine du processus réel, donc secondaires pour expliquer comment apparaît la gentrification, la demande et les préférences des consommateurs sont de première importance dans la détermination de la forme finale et du caractère des quartiers revitalisés. " (Smith, 1979, p. 540).

Smith conclut ainsi : "Les forces économiques ont plus stimulé la soit-disant renaissance urbaine que les forces culturelles. Dans la décision de réhabiliter les centres-villes, une préférence des consommateurs tend à dominer toutes les autres: la préférence pour le profit, ou plus précisément, pour un investissement financier solide. Il est fondamental que les gentrifieurs expriment cette préférence, puisqu'ils seraient très peu nombreux à entreprendre la réhabilitation s'ils s'attendaient à des pertes financières. Une théorie de la gentrification doit donc expliquer pourquoi la réhabilitation est profitable dans certains quartiers et pas dans d'autres. Quelles sont les conditions de profitabilité ? Les explications par la souveraineté des consommateurs ont considéré comme acquise la disponibilité de quartiers mûrs pour la réhabilitation, alors même que c'est ce point qui devait être expliqué.» (Smith, 1979, pp. 540-541).

Smith développe alors sa théorie du différentiel de loyer. Cette théorie est désormais bien connue et je ne peux y revenir dans ses détails. Il suffit de préciser que cette théorie situe la gentrification parmi des changements à long terme d'investissements et de désinvestissements dans l'environnement bâti, et met l'accent sur les rapports entre valeurs du sol et de la propriété, particulièrement sur la manière dont le désinvestissement rend possible un réinvestissement en capital. Smith montre qu'au $\mathrm{XIX}^{\mathrm{e}}$ siècle, la plupart des villes avaient un gradient de valeurs foncières classique, élevé au centre, et faible à la périphérie. Mais la suburbanisation des activités industrielles et de la population a fait diminuer les valeurs foncières des centres, et un fossé s'est creusé au cours des années de suburbanisation (1940-1960). Cette dévalorisation des centres a ensuite fourni la base de réinvestissements profitables.

41 Pour Smith, la clé est constituée par la relation entre prix du terrain et prix de la propriété bâtie. Quand la dépréciation des constructions existantes est suffisamment avancée, on en arrive à la situation où la rente foncière du site ou du quartier est plus faible que la rente foncière potentielle dans son meilleur usage. C'est le différentiel de loyer et, selon Smith, la gentrification ou le redéveloppement peuvent alors se produire quand ce différentiel est suffisant pour assurer un profit : «Lorsque le différentiel de loyer est suffisamment élevé, la gentrification peut se développer, dans un quartier donné, par 
différents acteurs, sur les marchés foncier et immobilier. Nous revenons alors aux rapports entre production et consommation, parce que les preuves empiriques suggèrent que le processus est initié, non par l'exercice de ces fameuses préférences du consommateur chères au coeur des économistes néo-classiques, mais par quelque forme d'action collective à l'échelle du quartier.» (Smith, 1979, p. 545).

Smith s'oppose fermement à toute explication de la gentrification formulée en termes de préférences des consommateurs individuels. En indiquant l'importance des prêts hypothécaires dans ce processus, il montre que «ces derniers constituent un préalable. Bien sûr, ces prêts doivent être faits par des consommateurs qui exercent des préférences. Mais ces préférences ne sont pas des nécessités, puisqu'elles sont créées socialement. » (Smith, 1979, pp. 545-546).

résume ainsi sa thèse : «La gentrification est le produit structurel des marchés foncier et immobilier. Les flux de capitaux dont le taux de profit est le plus élevé et les mouvements de capitaux vers les banlieues avec la dépréciation continue du capital des centres-villes, produisent en définitive la rente foncière. Lorsque cet écart se creuse suffisamment, la réhabilitation (ou, en ce cas, la rénovation) peut commencer à concurrencer les taux de retour disponibles ailleurs et le capital revient. » (Smith, 1979, p. 546). C'est la raison du sous-titre du texte de Smith, «le mouvement de retour du capital, non des gens, vers les centres".

Une évaluation de la théorie du différentiel de loyer de Smith

C'est une argumentation élégante et Smith avait raison d'essayer de déplacer l'accent des premières théories en faveur des préférences des consommateurs et de la demande vers la prise en considération de l'offre de propriétés gentrifiables et du rôle des emprunts immobiliers et du profit. Mais il est désormais clair qu'en dépit de l'importance de sa thèse du différentiel de loyer pour la compréhension du processus inégalitaire d'investissement, de désinvestissement et de réinvestissement dans l'environnement bâti, son rejet des explications alternatives, notamment du rôle de la nouvelle classe et de ses caractéristiques de consommation et de culture, et son refus d'accorder le moindre rôle aux acteurs individuels limitent l'intérêt de sa thèse. Ainsi dans son explication de la gentrification, Smith n'accorde aux gentrifieurs individuels que le rôle de serviteurs passifs des besoins du capital.

Il est judicieux de commencer par le refus qu'oppose Smith à la théorie de la demande de consommation et à la thèse postindustrielle de Ley. Smith reconnaît que seule la thèse postindustrielle de Ley est suffisamment large pour rendre compte de la gentrification à l'échelle internationale, mais il la rejette comme contradictoire. Si les préférences individuelles changent simultanément, il ne peut s'agir de préférences individuelles, ou alors les contraintes exogènes sont suffisamment fortes pour faire entrer de force ces préférences dans le même moule. Il y a quelque vérité dans le second argument. Les préférences de consommation n'émergent pas du néant. Elles sont, pour une part, socialement créées, manipulées et mises en forme, et elles le sont nécessairement sur la base d'options et de contraintes qui ne reflètent pas toujours des choix purement individuels. En revanche, Smith se trompe lorsqu'il estime que, pour que le concept de préférence individuelle soit valide, les individus doivent faire des choix différents dans différents pays. Si dans des pays différents des groupes similaires choisissent des options similaires, il est peu surprenant qu'il y ait des résultats similaires. Mais ceci ne veut pas dire que les individus sont totalement déterminés dans 
leurs choix, comme Smith semble le conclure (1979, p.540), ou que toutes les préférences sont créées socialement.

La solution proposée par Smith tend à redéfinir les préférences en termes de "préférences sociales collectives", mais elle ne rend pas compte des origines de ces préférences sociales collectives. Cette solution ne fait que déplacer le problème, de l'explication des origines des préférences à une échelle idéologiquement plus acceptable, mais à un niveau théorique mystérieux. Il faut aussi remarquer que seule une minorité de personnes décide de vivre dans les centres-villes et de devenir des gentrifieurs. Beaucoup d'autres décident d'aller vivre en banlieue. Aussi reste-t-il à expliquer pourquoi certaines personnes font une chose et pourquoi d'autres en font une autre. Ceci ne peut être expliqué en termes de flux de capitaux, de mouvements de désinvestissement-réinvestissement. Bien que le processus de gentrification implique des flux de capitaux, il implique aussi des personnes et c'est le talon d'Achille de la thèse de Smith, fondée sur le seul aspect de l'offre.

Non seulement Smith relègue les préférences des consommateurs et la demande à un rôle subalterne en faveur de la production de l'espace résidentiel, mais il considère aussi qu'une des raisons qui expliquent que certains quartiers valent la peine d'être redéveloppés et d'autres non, constitue certainement le cœur d'une théorie de la gentrification. Smith a raison dans la mesure où les explications en termes de demande tenaient pour acquise la disponibilité de quartiers mûrs pour la gentrification. Mais, comme nous le verrons, Smith tombe dans un piège presque similaire en convenant de l'existence d'un groupe de gentrifieurs et des conditions de la demande. Il fait l'hypothèse que si les conditions de profit étaient favorables, alors la gentrification (ou la rénovation) aurait lieu et les gentrifieurs potentiels seraient enclins à jouer un rôle dans le processus de revalorisation. Ce n'est que plus tard qu'il tenta de rectifier cette lacune, elle-même fruit de sa tendance à postuler que la demande est secondaire par rapport à l'offre dans l'explication de la gentrification.

Smith a raison d'insister sur le rôle décisif des prêts d'accession à la propriété dans la restructuration urbaine, comme Harvey (1974), Williams (1976, 1978), Boddy (1976) ; Dingemans (1979), Wolfe et al. (1980), Hamnett et Randolph $(1986,1987)$ l'ont aussi fait. Bien que l'absence de prêts hypothécaires rende la gentrification impossible, sauf à grande échelle, leur seule existence ne crée pas pour autant la gentrification. Les prêts hypothécaires sont une condition nécessaire, mais non suffisante, de la gentrification à grande échelle. De même il est incorrect de postuler que, "bien que les prêts hypothécaires doivent être pris par des consommateurs exerçant une sorte de préférence... les préférences peuvent être créées socialement » (Smith, 1979, p. 546).

C'est un point correct, mais Smith sous-entend que toutes les préférences sont créées socialement, ce qui n'a pas de sens. On ne vérifie pas non plus empiriquement le postulat selon lequel "le processus n'est pas initié par l'exercice des préférences des consommateurs individuels, mais par quelque forme d'action sociale collective menée à l'échelle $d u$ quartier» (Moore, 1982, p.545). Smith tend constamment à nier le rôle des gentrifieurs individuels en faveur des acteurs sociaux collectifs; cela apparaît sans ambiguïté lorsqu'il identifie les trois types de promoteurs qui opèrent typiquement dans les quartiers en gentrification. C'est-à-dire :

50 (a) les promoteurs professionnels qui achètent les propriétés, les remettent en état et les revendent pour le profit; 
51 (b) les promoteurs propriétaires-occupants, qui achètent et réhabilitent la propriété pour l'habiter ensuite ; sur l'importance centrale des intérêts des producteurs et le rôle secondaire du choix des consommateurs, les ménages sont eux-mêmes l'une des forces les plus importantes et les plus appropriées dans la production des quartiers gentrifiés. Ce n'est qu'en les classant comme promoteurs qu'il est capable de circonvenir l'intrusion fâcheuse de la rénovation individuelle pour la consommation dans sa thèse où prédominent les producteurs. Dans la mesure où les producteurs/consommateurs individuels jouent un rôle-clé dans le processus de gentrification (et c'est certainement vrai à Londres), la distinction de Smith entre production et consommation est artificielle et il n'arrive pas à expliquer d'où viennent les gentrifieurs-promoteurs individuels et pourquoi certains individus plutôt que d'autres deviennent des gentrifieurs. Dans l'analyse de Smith, les individus semblent gentrifier à cause du différentiel de loyer, quels que soient leurs caractéristiques, leurs goûts et leurs demandes. Mais comme D. Rose (1984) le montre : "Les gentrifieurs ne subissent pas simplement un processus déterminé indépendamment d'eux. Leur constitution, en tant que types de travailleurs et comme groupe social est un élément aussi crucial dans la production de la gentrification que dans la production de l'habitat qu'ils occupent. Ils peuvent faire ou ne pas faire que le processus ait lieu dans des situations particulièrement contingentes. " (Rose, 1984, p. 56).

Ctte proposition de D. Rose accuse le caractère économiciste et déterministe de la théorie du différentiel de loyer de la gentrification et de sa trop grande insistance sur la production des quartiers gentrifiables. On ne saurait trop insister sur le fait que la gentrification ne se produit pas indépendamment des gentrifieurs individuels. Quoique le différentiel de loyer puisse ne pas être nécessaire pour que la gentrification survienne, il n'est pas suffisant non plus pour qu'elle se développe. En effet, la théorie du différentiel de loyer ne dit rien des raisons pour lesquelles la gentrification se produirait plutôt qu'une autre forme de rénovation ou de réhabilitation. La théorie du différentiel de loyer est donc substantiellement sous-déterminée. La gentrification peut ne pas apparaître là où le différentiel de loyer existe ; c'est un phénomène contingent. La gentrification peut apparaître, tout comme la rénovation, la détérioration ou l'abandon.

Étant donné que le différentiel entre les loyers de base potentiels et réels est anticipé à partir du loyer de base potentiel, D. Smith parle très peu des processus par lesquels ces loyers de base potentiels se forment. Il est possible, par exemple que dans les quartiers en gentrification, le loyer de base potentiel soit en partie le résultat de la demande de gentrifieurs potentiels (Moore, 1982). Comme Munt (1987) l'expose: "Parce que les gentrifieurs peuvent choisir parmi de nombreuses localisations résidentielles à l'intérieur des centres-villes, l'ampleur du différentiel de loyer dans certains sites dépend de leur attractivité, donc de la demande, qui est absente de la théorie marxiste de la gentrification. » (p. 177). Ley va plus loin en posant que le différentiel de loyer n'est même pas un élément nécessaire de la gentrification. Dans sa perspective, le potentiel de profit et la capacité des gentrifieurs à surpasser les usagers existants ou potentiels pour les sites urbains 
centraux suffisent. D. Ley estime aussi que la plupart des promoteurs ont une aversion du risque et n'oseront pas choisir tel quartier, tant qu'il n'est pas prouvé que la demande existe : «Du point de vue des promoteurs, la demande est le strict minimum. En bref, le capital suit la demande, ce qui n'empêche pas la manipulation des marchés locaux provoquant par exemple la ruine d'un ilot ou le fait que la demande soit produite par des contextes économiques plus larges. » (1990, communication personnelle).

Deux recherches empiriques récentes ont analysé ces difficultés liées à la thèse du différentiel de loyer. Clark (1988) a prouvé l'existence d'un différentiel de loyer dans son travail inédit sur l'évolution des valeurs foncières et immobilières à Malmö, Suède. Néanmoins, il considère que cela était théoriquement explicable, soit dans les termes de la théorie néoclassique de Marshall, soit dans ceux de la théorie marxiste de Smith. Le différentiel de loyer n'était en aucun cas un déterminant de la gentrification ou une explication complète de ce processus. En fait le redéveloppement plutôt que la gentrification s'est manifesté partout à Malmö. Ainsi Clark rejette l'idée "d'un développement prédéterminé, dont le facteur premier serait les besoins du capital et dont le différentiel de loyer serait le mécanisme à détente. L'action des agents ayant des intérêts politiques ou économiques et celle d'individus s'intéressant à leur propre logement sont essentielles aux histoires particulières qui se déroulent en un lieu » (Clark, 1988, p. 244.)

Dans son travail sur Adélaïde (Australie du Sud), Badcock (1989) apporte des preuves convaincantes qu'un différentiel de loyer important s'était développé dans la City et dans quelques-unes des banlieues résidentielles victoriennes situées dans sa proximité. Une importante gentrification était apparue, annulant le différentiel de loyer. Mais il concluait aussi que "nulle part les processus responsables de ce différentiel de loyer ne sont aussi nets que ce que prétend Smith" (Badcock, 1989, p.132). Il montre que la gentrification était la troisième meilleure réponse du capital aux conditions existant à Adélaïde et qu'elle était, de plusieurs manières, une stratégie d'investissement suboptimale (p.133). En d'autres termes, la gentrification n'était pas le résultat inévitable du différentiel de loyer.

Les résultats de ces deux recherches démontrent que l'existence d'un différentiel de loyer n'est pas une condition suffisante pour qu'apparaisse la gentrification. Au contraire, l'existence d'un différentiel de loyer peut mener à différents résultats, dont la rénovation ou la poursuite de la dévalorisation. Plus généralement, il apparaît que la théorie de Smith est valide dans la mesure où elle explique l'existence de quartiers urbains où apparaît la gentrification. Mais elle ne mentionne rien des raisons pour lesquelles la gentrification tend à apparaitre dans certaines villes et non dans d'autres. De même il n'aborde pas la question des caractéristiques et des origines des gentrifieurs eux-mêmes, et les raisons pour lesquelles ils se tournent vers la gentrification plutôt que la suburbanisation. Cette théorie n'en apporte pas moins une contribution majeure comme analyse des cycles d'investissement et de désinvestissement dans l'environnement bâti, mais son pouvoir d'explication d'autres aspects de la gentrification est limité.

La théorie de Smith ne pouvait traiter de ces autres questions principalement parce qu'étant centrée sur la production de l'environnement bâti, elle était "limitée à la spécification des préconditions pour la production de logements gentrifiés sans considération de la production de gentrifieurs, c'est-à-dire des occupants de tels logements» (Rose, 1984, p.51). Parce que Smith a centré son explication sur la production du différentiel de loyer et confondu, puis rejeté définitivement comme 
éléments de la thèse des "préférences », les changements dans les structures d'emploi, dans les comportements démographiques et dans les processus de reproduction sociale, il a ignoré des changements matériels essentiels influençant la production des gentrifieurs et mis sur le même pied les explications matérialistes et le différentiel de loyer.

La production des gentrifieurs et leurs choix de localisation

61 Au début des années 1980, la théorie de Ley de l'effet des changements de la division sociale et spatiale du travail, des structures d'emploi et de la montée d'une nouvelle classe moyenne sur la gentrification a été suivie de différentes manières par plusieurs autres chercheurs. Ils avaient établi des liens théoriques entre les changements dans ces restructurations sociales et spatiales et la production des gentrifieurs selon les termes de D. Rose (1984). De telles relations ont été notamment établies par Mullins (1982), qui estime que des changements considérables se sont produits dans les centresvilles en Australie. Le déclin dans les centres-villes de l'industrie et de la classe ouvrière qualifiée qui y résidait a été accompagné par l'émergence de centres d'affaires propre au capitalisme monopolistique et aux couches moyennes salariées qu'il emploie. Mullins liait ces changements à la gentrification, insistant sur le fait que "puisque la classe ouvrière d'une forme antérieure de centre-ville vivait dans ces quartiers pour des raisons d'emplois centrées sur l'industrie manufacturière, "la force de travail diplômée" est en train de venir s'installer dans les centres-villes uniquement pour des raisons de consommation" (pp. 45-46). Mais, comme Mullins le note, «le développement de l'emploi de bureau ne peut pleinement expliquer celui des travailleurs très qualifiés résidant en centre-ville, simplement parce que la majorité de ces salariés résident dans les banlieues et se déplacent quotidiennement. D'autres processus doivent aussi avoir joué dans ce développement résidentiel » (Mullins, 1982, p.53). Une relation similaire a été établie par Moore (1982) qui estime que «la gentrification représente le processus grâce auquel une importante fraction de la nouvelle classe est en train de se constituer une identité résidentielle congruente avec son identité sociale, dans un contexte global où les centres-villes deviennent de plus en plus des villes de cols blancs."

Mullins insiste sur le rôle-clé de la production et de la consommation de services artistiques particulièrement orientés vers les loisirs, qui sont produits et consommés par un petit nombre de travailleurs diplômés. Cette explication de la gentrification est liée à la production de gentrifieurs et à leurs exigences culturelles. Similaire à celle de la thèse de Ley, elle isole les raisons pour lesquelles les membres de la nouvelle classe sont géographiquement concentrés dans les centres-villes : les besoins culturels et la concentration d'équipements culturels en centres-villes. La question de la localisation est d'une importance cruciale. Ce que Mullins a compris, c'est que la croissance d'une nouvelle classe moyenne ou d'une classe de services ne suffit pas à expliquer la gentrification. Une explication suffisante devrait en effet aussi rendre compte des raisons pour lesquelles certains membres de ce groupe résident dans les centres-villes plutôt qu'ailleurs (voir aussi Moore, 1982).

63 L'argument concernant le rôle-clé de la production de gentrifieurs potentiels a été développé par D. Rose (1984), qui estimait que : «Les travaux empiriques et théoriques des marxistes ont été principalement centrés sur ces aspects de la gentrification qui peuvent être directement liés au jeu de la loi de la valeur dans l'environnement bâti des villes capitalistes... Cela n'a pas seulement créé un fossé analytique, mais procédé aussi d'une erreur épistémologique d'une importance considérable. » (Rose, 1984, p. 52). 

entre gentrification et changements dans la restructuration sociale et spatiale des processus de travail et dans la reproduction de la force de travail et des individus. Ces liens ont été largement ignorés par les approches économicistes : selon elles, ou bien les processus sociaux sont explicables d'un point de vue économique, ou bien on les considère comme des épiphénomènes. De la même manière, Beauregard estimait que le seul différentiel de loyer n'est pas une explication fondée de la gentrification. Pour expliquer la gentrification, il faut commencer par isoler la présence de gentrifieurs, les agents nécessaires et les bénéficiaires du processus de gentrification, les directions prises par leur reproduction et par leur consommation (Beauregard, 1986, p. 41). des logements centraux peu chers n'est pas un phénomène nouveau et ne peut être expliquée simplement par le différentiel de loyer/salaire. Deuxièmement, "les gentrifieurs sont souvent, quoique rarement de manière exclusive, les "agents" du processus de gentrification et fournissent ainsi les motivations et les aspirations qui le forment." Troisièmement, le processus cesse d'exister sans ce groupe. Différents types de logements peuvent être réhabilités, mais comme les caractéristiques des gentrifieurs sont largement similaires, bien que les quartiers dans lesquels ils résident soient différents, «la gentrification est définie par la présence de gentrifieurs " (Beauregard, 1986, p. 41).

Cet argument diffère radicalement de celui proposé par N. Smith. La priorité causale est exactement inverse. Là où $\mathrm{N}$. Smith postulait l'existence de gentrifieurs potentiels et voyait la production de quartiers appropriés comme clé du processus, Beauregard considère que les gentrifieurs sont la clé d'explication du processus. La gentrification sans gentrifieurs n'existe pas. Comme D. Ley et P. Mullins, R. Beauregard insiste sur le rôle crucial joué par les changements dans les structures industrielles et dans les structures d'emploi et indique que c'est au sein de la «fraction urbaine des cadres moyens et supérieurs » que se situent les gentrifieurs. Sur les traces de D. Rose (1984), P. Mullins (1982), P. Moore (1982) et P. Williams (1984), R. Beauregard estime que: "Pour expliquer les raisons pour lesquelles ces cadres moyens et supérieurs... restent dans les centres des villes et entreprennent aussi la gentrification, nous devons nous éloigner de la sphère de la production et mettre l'accent sur leurs activités de reproduction et de consommation... La question étant de savoir quel intérêt il y a, mise à part la proximité du lieu de travail, à posséder une résidence urbaine, qui corresponde particulièrement aux activités de consommation et de reproduction de cette fraction des actifs ?» (1986, p. 43).

différentiel de loyer ne fournit qu'une des conditions nécessaires de la gentrification et aucune des conditions suffisantes..." Beaucoup de quartiers des agglomérations centrales ont des différentiels de loyer bien supérieurs à ceux des quartiers qui se gentrifient. Aussi cette théorie ne peut expliquer aisément pourquoi Hoboken se gentrifie, mais non Newark (1986, p. 39).

C'est un point important qui affaiblit considérablement les positions de N. Smith. Pour résumer, il est clair que l'existence d'un ensemble de gentrifieurs potentiels appartenant à la nouvelle classe moyenne est un préalable nécessaire pour que la gentrification ait lieu, de même que la présence de quartiers et de logements potentiellement gentrifiables. Mais aucune de ces deux conditions ne suffit à faire apparaître la gentrification. Il faut qu'en outre une partie du groupe des cadres moyens et supérieurs souhaite vivre dans les centres-villes, mais aussi que les équipements et 
environnements appropriés existent. Sans ces préalables, il est très peu probable que la gentrification se développe, quelles que soient l'action des promoteurs et la disponibilité d'emprunts hypothécaires.

La tentative de Smith pour intégrer la consommation dans la gentrification

En 1986, N. Smith tenta de replacer la théorie du différentiel de loyer dans un cadre d'analyse plus large de la gentrification, incluant la désindustrialisation des économies capitalistes, le poids croissant des cols-blancs, ainsi que des changements dans la démographie et dans les modes de consommation. Son approche s'élargit à la suite de cette tentative et $\mathrm{N}$. Smith précise que "bien que les tentatives précédentes d'explication aient tendu à se raccrocher à l'une ou l'autre de ces lignes directrices, on ne pouvait pas considérer l'une sans l'autre» (p. 21). Il le dévoile en ces termes: «les changements dans les schémas démographiques et dans les préférences en matière de style de vie ne sont pas totalement hors de propos, mais... l'enjeu principal de ces thèmes semble plus se concentrer sur l'analyse de l'apparence extérieure de la plupart des opérations de restructuration urbaine, que sur l'explication du fait même de la transformation urbaine. Compte tenu des mouvements de capitaux dans le centre-ville et de l'importance accordée aux fonctions de direction, d'administration-gestion, aussi bien qu'aux autres fonctions d'encadrement, les changements démographiques et de style de vie... permettent d'expliquer pourquoi des restaurants Tarte Julie proliferent plutôt que des cafétérias Casino, des boutiques de vêtements à la mode et des épiceries fines plutôt que des modestes petites surfaces. »(Smith, 1986, p. 31).

Dans cette perspective, l'influence de la "consommation " se réduit au choix de la couleur et du dessin du glaçage sur le gâteau de la restructuration urbaine et de la gentrification. Cette vision reste indifférente aux arguments avancés par Moore, Beauregard et Ley sur le rôle fondateur de la culture et de la consommation dans le processus qui voit la classe montante gentrifier les centres-villes au lieu de s'installer en banlieue. Bien que Smith accepte qu'il soit important d'expliquer le rôle des changements dans la structure de production et l'évolution de la division spatiale du travail dans la production des cadres moyens et supérieurs, il n'explique pas pourquoi une partie de ce groupe s'installe en centre-ville. Et quand il aborde le rôle des gentrifieurs, il rejette résolument l'hypothèse qu'ils pourraient jouer un rôle important dans ce processus: "à l'instar de la frontière se développe le mythe selon lequel la gentrification est due à des pionniers individuels et des fermiers dont l'équité, l'audace et la perspicacité montrent le chemin à ceux qui parmi nous sont les plus timides. Mais il est clair que là où s'aventurent ces pionniers urbains, les banques, les agences immobilières, l'État ou d'autres acteurs collectifs de la vie économique les ont précédés. »(Smith, 1986, pp. 18-19).

Or ce point n'est confirmé par aucune preuve pour les cas de Londres et New York (Zukin, 1987), qui montrent que les pionniers individuels jouent un rôle initiateur important, même s'ils peuvent être souvent pris de vitesse par les banques, les promoteurs et les agents immobiliers. Munt (1987) estime que dans Battersea, un processus graduel d'infiltration par les gentrifieurs a précédé des opérations plus massives de rénovation (p. 1177). Il existe de fortes preuves contredisant Smith et montrant que les pionniers urbains ont déjà exploré les lieux où les acteurs économiques collectifs s'aventurent (Goetze, 1979).

En 1987, dans un important article intitulé «Des Yuppies et de la gentrification: la gentrification, le changement social et le rêve urbain", Smith tente de lier les arguments de la restructuration urbaine et de la consommation. Considérant d'abord la question de l'existence d'une nouvelle classe moyenne, Smith admet qu'il y a eu une 
indéniable transformation des structures d'emploi, les cadres moyens et supérieurs des secteurs en expansion étant largement représentés parmi les gentrifieurs (Smith, 1987, p. 154). Mais il estime que ceci ne prouve pas l'existence d'une nouvelle classe moyenne en termes marxistes (c'est-à-dire liée à la propriété et au contrôle des moyens de production). Cet argument est correct, mais dans la mesure où la thèse de la restructuration sociale met en priorité l'accent sur le changement des structures d'emploi, et non sur la validité théorique des catégories de classe marxistes, elle est tout à fait incorrecte, et Smith semble accepter l'existence d'une nouvelle « classe » en termes empiriques, voire en termes de théorie marxiste des classes sociales. Comme il l'explique : "Certes, les structures d'emploi ont considérablement changé, une restructuration sociale profonde est en cours... et elle altère la structure sociale de la société. De la même manière, cette restructuration sociale est lourdement impliquée dans le processus de gentrification. »(Smith, 1987, p. 161).

Mais, bien que Smith reconnaisse que les nouvelles formes de travail revêtent une importance primordiale dans les processus de restructuration sociale lorsqu'il s'agit d'expliquer la gentrification, il avance qu' «ils posent aussi des risque intrinsèques avec eux. Si la gentrification doit être d'abord et avant tout expliquée par l'émergence d'un nouveau groupe social... il devient alors difficile de ne pas souscrire à une sorte de modèle de préférence des consommateurs, même atténué. Comment ce nouveau groupe social pourrait-il entraîner la gentrification, sinon en demandant des équipements et une localisation spécifiques de leurs logements sur le marché?» (Smith, 1987a, p. 163).

74 Les craintes de Smith sont très claires et le poussent à résoudre la difficulté qu'il y a à accepter l'existence d'un nouveau groupe social sans lui donner un rôle clé dans le processus de gentrification. Sa "solution » est ingénieuse et met en lumière ce qui est peut-être le problème-clé dans l'explication de la gentrification, c'est-à-dire sa manifestation spatiale: «Il est indiscutable que la demande peut, à certains moments, en particulier quand elle change considérablement, altérer la nature de la production. Mais le mystère de la gentrification n'amène pas à expliquer d'où vient la demande des couches moyennes, mais plutôt à expliquer le problème essentiellement géographique des raisons pour lesquelles les centres-villes, qui n'avaient pu satisfaire la demande des classes moyennes pendant des décennies, semblent désormais le faire de manière aussi satisfaisante. En effet, si la structure de la demande a changé, nous avons besoin d'expliquer pourquoi ces demandes nouvelles se concentrent à nouveau dans les centres-villes. » (Smith, 1987a, pp. 163-164).

La discussion menée par Smith est fascinante. Ayant admis que la demande puisse altérer la nature de l'offre, il en évite alors les conséquences : il considère que l'énigme de la gentrification ne porte pas sur l'origine de la demande, mais sur la forme géographique qu'elle revêt. Cette question est fondamentale pour l'explication de la gentrification. Mais il s'agit seulement de la moitié de l'enjeu. Le mystère de la gentrification porte à la fois sur l'origine de la demande de la classe moyenne et sur son émergence dans les centres-villes. Cependant Smith identifie la deuxième question comme la question-clé : "Sans aucun doute, une centralisation continue, voire accélérée des fonctions de gestion-administration rend un domicile central plus désirable pour une importante partie des classes moyennes. Mais ces arguments correspondent-ils à une explication de l'inversion géographique des règles de localisation de l'habitat par une proportion importante d'hommes et de femmes des couches moyennes?... L'argument selon lequel la restructuration sociale est le principal moteur de la gentrification est substantiellement sous-déterminé." (Smith, 1987, p. 164). 
76 La thèse de Smith selon laquelle la restructuration sociale n'est pas une explication adéquate de la gentrification est correcte. Mais, comme nous l'avons vu, les tenants de la thèse de la restructuration sociale ne sont pas d'accord avec lui. Au contraire, ils insistent tous sur le rôle décisif des conditions, à la fois culturelles et de consommation, propres à une fraction de la nouvelle classe, et estiment que ces conditions sont remplies par une localisation intra-urbaine. C'est une relation causale entre la production d'une nouvelle force de travail de cadres moyens et supérieurs dont une partie a les caractéristiques culturelles et de consommation requises, et la création de gentrifieurs potentiels. Il y a deux étapes dans cette argumentation, et non une seule, mais Smith n'admet que la première et élimine la seconde. Il n'est donc pas surprenant qu'il conclue: "Je voudrais défendre l'analyse du différentiel de loyer... non en tant qu'explication définitive ou complète de la gentrification, mais en tant que pièce maîtresse de toute théorie de la gentrification. Ce sont avant tout les schémas historiques d'investissement et de désinvestissement capitalistique dans les centres des villes qui établissent l'opportunité (non la nécessité) de l'inversion spatiale.» (Smith, 1987a, p. 165).

77 Cette proposition est en net retrait par rapport à la position initiale de Smith et présuppose ce que Badcock $(1989$, p.126) appelle un "considérable relâchement des postulats originels de la théorie ». Smith semble désormais considérer la théorie du différentiel de loyer comme une clé qui traduit des processus plus généraux, c'est-àdire la production de gentrifieurs dans une inversion spatiale. Mais l'argument de Smith selon lequel le différentiel de loyer est la clé de voûte de toute théorie de la gentrification est une prétention excessive. Comme Smith le précise, le différentiel de loyer établit l'opportunité, non la nécessité d'une inversion spatiale. Le différentiel de loyer peut apporter les moyens, mais il ne peut apporter un motif à la gentrification. Pour cela nous avons besoin de regarder ce qui est, pour Smith, le cœur de l'obscurité : les préférences de localisation, les styles de vie et la consommation.

78 Comme Smith juge inacceptable tout accent mis sur les styles de vie et sur la consommation des individus, en 1987 il mit l'accent sur une possibilité d'intégrer les arguments du côté de la consommation et de la production dans les termes d'une analyse historique de la restructuration sociale. Ceci impliquait le rejet des idées de Ley sur le postindustrialisme comme "une abstraction empirique étroite... incapable de soutenir la moindre analyse théorique " (Smith, 1987a, p. 166), tout en réinterprétant la substance de l'argument de la société de consommation dans les termes de l'analyse régulationniste d'Aglietta. Smith soutient que, tandis que le régime intensif d'accumulation commençait à se décomposer dans les années 1970 et 1980, un changement s'est opéré vers un nouveau régime d'accumulation (postfordiste), associé non avec la production et la consommation de masse, mais avec une production et une consommation différenciées. Dans ce nouveau régime d'accumulation, l'accent porte sur la différenciation du produit et les marchés à niche. La gentrification est expliquée dans ces termes comme résultant des désirs des gentrifieurs de se différencier euxmêmes d'autres groupes sociaux. Comme Smith le note: "c'est cette question de différenciation culturelle dans un marché de masse qui est plus pertinente pour la gentrification. La gentrification est une redifférenciation du paysage culturel, social et économique... la gentrification et le mode de consommation qu'elle engendre sont partie intégrante de la constitution de classe. Elles sont une partie des moyens mis en ceuvre par les individus appartenant à la nouvelle classe moyenne de se distinguer de la bourgeoisie au-dessus d'eux et de la classe ouvrière au-dessous » (Smith, 1987, pp. 167-168). 

consommation postindustrielle. Mais l'interprétation de Smith de la consommation et son rôle dans la gentrification est, à l'évidence, très différente de celle suggérée par Ley et par d'autres. En mettant l'accent sur l'importance de la consommation dans le cadre de l'accumulation capitaliste, il a tenté de circonvenir les dangers inhérents au fait de donner aux gentrifieurs individuels un rôle-clé dans le processus de gentrification. Mais, mises à part de telles différences, le fait que Smith ait dû entreprendre cette réinterprétation est indicative des limites de la théorie du différentiel de loyer et de l'opposition fondamentale de Smith à toute concession sur le rôle des individus dans la constitution de leur environnement. Cependant le plus loin que Smith puisse aller est d'accepter le rôle des acteurs sociaux collectifs et les conditions fonctionnelles de la consommation différenciée dans le nouveau mode de régulation. Ce n'est pas que Smith refuse d'attribuer à l'initiative individuelle la primauté, mais c'est qu'il semble refuser d'accepter son existence autrement que de manière superficielle. Son opposition à toute forme d'explication par l'action (agence) fait de lui un structuraliste pour lequel l'action individuelle est réduite au rôle d'ombre scintillante jetée par les lumières de l'incendie du capital.

Vers une théorie intégrée de la gentrification

Il a été montré que les deux thèses, celle de la restructuration sociale, associée à Ley, et celle du différentiel de loyer, avancée par Smith, sont des tentatives partielles d'explication de la gentrification. L'approche de Ley est centrée sur les changements dans la division sociale et spatiale du travail, les changements dans les structures d'emploi, la création des demandes culturelles et environnementales et leur traduction dans le marché du logement par un pouvoir d'achat accru de la nouvelle classe. Il tenait pour acquise l'existence de quartiers potentiellement adéquats pour la gentrification et voyait le processus principalement en termes de demande sur le marché du logement.

81 Smith, pour sa part, a mis l'accent sur la production de logements gentrifiables par le mécanisme du différentiel de loyer. Il tenait pour acquise l'existence d'une demande de gentrifieurs potentiels et ignorait les raisons pour lesquels un segment de la nouvelle classe moyenne optait pour un habitat en centre-ville. Mullins, Moore, Beauregard et Rose ont débattu de la production de gentrifieurs: leurs caractéristiques sociales et culturelles étaient d'une importance cruciale pour la compréhension de la gentrification. Sans eux, le processus ne peut se développer. Mais la gentrification n'est pas simplement issue de changements dans la division sociale et spatiale du travail, aussi radicaux soient-ils. Il est aussi nécessaire qu'existent une préférence géographique en faveur des centres-villes ou des quartiers de logements spécifiques qui les composent, ainsi qu'une offre de quartiers et de logements gentrifiables définie non seulement en termes de différentiel de loyer, mais aussi en termes d'agrément ou d'attrait pour les gentrifieurs potentiels (Munt, 1987, pp. 1195-1196).

Il y a quatre conditions pour que la gentrification se produise de façon relativement ample. Les trois premières concernent respectivement l'offre de logements situés dans des quartiers propices à la gentrification, l'offre de gentrifieurs potentiels, et l'existence d'environnements attractifs dans les centres-villes. Ces conditions comprennent les éléments de l'équation nécessaires du côté de l'offre. La quatrième condition, décisive, fait intervenir une préférence culturelle pour la résidence en centre-ville d'actifs travaillant dans certains segments du secteur tertiaire. Une large gamme de résultats est possible selon la combinaison de ces quatre conditions. 
83 dégage de ce schéma est que la gentrification n'apparaît que sous une seule de ces combinaisons de circonstances. Aucune des autres combinaisons ne conduit à la gentrification, bien que Ley indiquerait qu'elle pourrait se produire sans un différentiel de loyer, tant que la nouvelle classe a le pouvoir d'achat pour déplacer ou remplacer les autres usagers du sol.

Tableau $\mathrm{n}^{\circ} 1$ : Schéma des conditions de la gentrification

\begin{tabular}{|l|l|l|}
\hline Le différentiel de loyer & existe & n'existe pas \\
\hline \hline Pas de gentrificateurs & Pas de gentrification & Pas de gentrification \\
potentiels & & \\
\hline \hline L'offre de gentrifieurs & & \\
potentiels existe & & \\
\hline \hline Pas de demande & Pas de gentrification & Pas de gentrification \\
pour le centre-ville & & \\
\hline \hline Préférence pour le centre & Gentrification & Gentrification \\
par une fraction & & \\
de la nouvelle classe & & \\
\hline
\end{tabular}

Mais il s'agit simplement d'une classification des circonstances. Elle n'offre pas, en tant que telle, de fondements pour une théorie de la gentrification. Et comme nous allons le voir, la question-clé d'une telle théorie est celle du point de départ. Il est inadéquat de considérer que la gentrification est le résultat d'une combinaison de circonstances sans essayer d'assigner quelque priorité théorique à ces circonstances. Je ne doute pas que - ainsi que l'a revendiqué Beauregard - «l'explication de la gentrification commence avec la présence des gentrifieurs" et que la "gentrification est définie par la présence de gentrifieurs» (Beauregard, 1986). Mais cela ne signifie pas que la culture et la consommation aient la place principale dans l'explication de la gentrification. Comme Ley, Mullins et d'autres l'ont indiqué, le point de départ adéquat est constitué par les changements dans les structures de production et dans la division sociale et spatiale du travail, qui ont conduit à la désindustrialisation des économies capitalistes avancées et à la croissance du secteur des services. Ces précisions ont à leur tour induit l'expansion rapide d'une nouvelle classe moyenne, liée à la concentration des fonctions-clés financières, juridiques et autres, dans un nombre relativement restreint de grandes villes telles que New York, Paris et Londres et dans nombre d'autres grandes agglomérations comme Vancouver, Toronto, Sydney et San Francisco. C'est dans ces villes que la gentrification a été la plus marquée.

L'explication de la gentrification doit ainsi commencer par les facteurs de la production et de la concentration des fractions-clés de la classe de service dans un certain nombre de métropoles. Ces processus ont engendré le groupe de gentrifieurs potentiels. Aussi l'accent doit-il d'abord être mis sur l'explication de l'expansion de ce groupe-clé. Cette 
explication n'est pas fondée sur la consommation, mais sur les changements dans les structures de production et dans la division sociale et spatiale du travail dans les pays capitalistes avancés. Il est ensuite nécessaire d'expliquer pourquoi la gentrification se produit dans certaines villes et non dans d'autres. Comme nous l'avons vu, deux conditions sont nécessaires. La première est qu'il est nécessaire d'avoir une offre de propriétés gentrifiables dans les centres-villes. C'est là où la théorie du différentiel de loyer entre en scène, expliquant pourquoi une offre de propriétés dévaluées situées en centres-villes existe, résultant d'un mouvement antérieur de suburbanisation et de décentralisation. La valeur potentielle de ces propriétés est supérieure à sa valeur courante. Mais comme nous l'avons vu, l'existence d'un différentiel de loyer ne conduit pas nécessairement à la gentrification. Sans l'existence d'un groupe de gentrifieurs potentiels et une offre de prêts d'accession à la propriété, la gentrification ne se produira pas, aussi élevé le différentiel de loyer et aussi grand le désir des promoteurs de la créer soient-ils. Là où ce stock de logements n'existe pas en quantité suffisante dans les centres-villes, comme à Dallas, Phœnix et d'autres villes du sud et de l'ouest des États-Unis, la gentrification peut être très limitée, aussi importante la classe de service soit-elle. Dans des villes plus anciennes du nord-est des États-Unis, comme Baltimore, Philadelphie et Washington D.C., les maisons du XIX ${ }^{\mathrm{e}}$ siècle, pour la plupart très dégradées, dévalorisées et hébergeant la classe ouvrière et les minorités constituent une offre abondante. Dans de telles villes, la gentrification a progressé à grands pas.

En deuxième lieu, il faut qu'existe une demande effective pour des propriétés localisées en centre-ville de la part de gentrifieurs potentiels. Cela peut résulter de l'incapacité financière d'acheter une maison en banlieue ou, comme il en est plus souvent fait état, d'une préférence pour la vie dans les centres, à proximité des emplois et des équipements culturels et sociaux. Cette préférence dépend à son tour de la croissance des emplois de service dans le centre d'affaires et des changements démographiques et des styles de vie. Ces derniers ont notamment consisté en une entrée massive des femmes sur le marché du travail, une augmentation du nombre de célibataires et de couples biactifs sans enfants. Pour ces groupes aisés, les localisations résidentielles intra-urbaines offrent la proximité aux emplois, aux restaurants, aux arts et à d'autres équipements. Il n'est pas surprenant qu'une proportion élevée d'entre eux semble avoir opté pour la résidence en centre-ville. Sans cette demande effective, créée par l'attraction pour la vie en centre-ville, il est peu vraisemblable que la gentrification se produise, aussi importante l'armée de gentrifieurs potentiels soit-elle, et aussi large le différentiel de loyer soit-il.

Il faut donc prendre en compte trois ensembles de conditions, qui sont tous nécessaires et dont aucun n'est suffisant. Mais il est clair que, sur les plans logique et théorique, l'existence d'un groupe potentiel de gentrifieurs est primordiale, par rapport aux préférences de logement et de styles de vie d'une partie de la classe de service. Et, tandis que l'existence d'une offre de maisons adéquates situées en centre-ville est nécessaire pour que la gentrification se produise, l'existence d'un différentiel de loyer ne produirait pas, à lui seul, la gentrification. Il est donc difficile d'accepter la perspective de Smith, selon laquelle le différentiel de loyer est «la pièce maîtresse de toute théorie de la gentrification ». Aussi nécessaire soit-il, si la théorie de la gentrification a une pièce maîtresse, celle-ci doit être fondée sur la production de gentrifieurs potentiels. 
Conclusions

88 J'ai essayé de montrer que les débats sur l'explication de la gentrification ont été largement centrés sur le conflit entre les chercheurs qui estiment que la clé du problème tient dans les changements globaux des structures de production et de la division sociale et spatiale du travail, ou dans la concentration dans certaines métropoles d'une section de la nouvelle classe moyenne ou classe de service, ayant une composition démographique et des orientations culturelles et de consommation particulières. D'autre part, Smith a abondamment plaidé pour l'argument de l'investissement et du désinvestissement dans l'environnement bâti et pour une approche fondée sur la priorité donnée à la profitabilité. Ce conflit s'est manifesté de diverses façons - entre les explications menées en termes d' "offre » et de "demande », entre choix et culture contre capital, etc. Cependant j'avais montré que beaucoup de ces dualismes et polarités étaient plus apparents que réels, et ce que Smith appellerait le côté des " choix, consommation et culture " a en fait toujours eu un pied fermement planté dans les changements réels de la base matérielle de la production et de ses manifestations culturelles.

89 D'une certaine façon, le conflit a porté sur deux interprétations de la production - la première portant sur les changements dans la division sociale et spatiale du travail et sur la production des gentrifieurs, et la seconde prenant en compte la production de l'environnement bâti. Mais, encore récemment, Smith interprétait la première approche en termes de consommation, de culture et de choix individuels, et rejetait son apport. Smith a reconnu par la suite qu'il est important d'intégrer production et consommation, mais cette intégration reste formulée en termes d'un cadre de travail, qui, ou bien attribue la primauté aux questions de production, ou bien réintègre la consommation d'une manière collective non problématique. Smith conçoit l'action individuelle comme limitée, circonscrite. Certes, il accepte que les acteurs sociaux collectifs puissent engendrer la gentrification, mais non une multiplicité d'acteurs individuels. Si les critiques de la position de Ley ont été plus limitées, c'est en partie parce qu'il a atténué ses propos et été moins affirmatif dans ses options concernant la priorité théorique. On doit reconnaître qu'il a judicieusement saisi le rôle-clé d'un nouveau groupe de gentrifieurs potentiels avec leurs exigences. Il a péché par omission plus que par engagement. Ley a peu examiné l'offre de logements et le rôle des promoteurs/spéculateurs dans le processus. Ils sont considérés comme dérivant largement des demandes de la nouvelle classe.

90 Aujourd'hui Smith admet en partie que sa position sur la gentrification comme résultat structurel du foncier et du marché immobilier est discutable. Il ne faut pas pour autant considérer que la thèse du différentiel de loyer était fausse. Seulement le différentiel de loyer n'explique au mieux que la moitié du problème, voire moins. L'existence de logements peu chers et dévalorisés est un élément nécessaire, mais loin d'être suffisant, d'une explication. De la même manière, l'explication par la production de gentrifieurs potentiels, leur culture, leur consommation et leur reproduction, est nécessaire mais insuffisante. Une explication exhaustive de la gentrification doit nécessairement prendre en compte l'origine des gentrifieurs et les raisons pour lesquelles ils gentrifient, la façon dont sont produits les quartiers et les immeubles à gentrifier, et les liens entre ces deux ensembles de conditions. Et il est fort possible que, quel que soit le rôle des acteurs sociaux institutionnels et collectifs comme les agents immobiliers, les promoteurs et les sociétés d'emprunts hypothécaires, les acteurs-clés dans le processus 
de gentrification aient été les gentrifieurs eux-mêmes. Il faut accepter que l'action individuelle explique une bonne partie de la gentrification et chercher à intégrer la production et la consommation, non en termes de causes structurelles ou d'effets individuels, mais en termes d'action individuelle et de structures.

Parce que Smith a développé la théorie du différentiel de loyer, il s'est vigoureusement défendu, opérant des retraites tactiques et des concessions quand c'était nécessaire, mais recherchant essentiellement à s'assurer une place centrale dans l'explication de la gentrification. Mais tandis qu'il a accepté que les changements dans la division sociale et spatiale du travail et la concentration de l'emploi professionnel et directionnel dans les quartiers centraux d'affaires étaient primordiaux, et qu'il a tenté d'intégrer les modes de consommation des gentrifieurs dans ses théories, il l'a fait de telle sorte que cela devenait une exigence fonctionnelle du capitalisme tardif, plutôt qu'une reconnaissance $\mathrm{du}$ rôle des préférences et de l'action individuelle. Mais les interventions de Smith dans le débat sur la gentrification n'ont pas été contreproductives. Au contraire, ce n'est qu'en défiant les théories des choix et préférences et en plaidant pour une alternative logique et cohérente qu'il a amené aussi loin le débat sur les explications possibles. Précisément parce que Ley et Smith ont été les pionniers de théories et d'interprétations radicalement différentes de la gentrification, il a été possible de faire progresser notre compréhension du processus en considérant comment les deux interprétations partielles s'ajustent entre elles. S'il a été montré que ces travaux sont limités sur certains points-clés, c'est le prix payé par les pionniers théoriques. Aucun d'entre eux n'a reconnu l'éléphant de la gentrification d'emblée, mais chacun a identifié une partie clé de son anatomie alors que d'autres chercheurs ont été capables d'intégrer ensemble une explication plus complète. Clark conclut ainsi : "Nous devrions cesser de poser cette question unidimensionnelle: "Quelle théorie de la gentrification est juste, la théorie du différentiel de loyer, la théorie de la restructuration postindustrielle, la théorie de la demande d'équipements par les consommateurs ou la théorie institutionnaliste ?" Il faut commencer à se demander "S'il existe des preuves pour chacune de ces théories, pouvons-nous parvenir à la compréhension des chemins par lesquels elles se tiennent dans une relation logique de complémentarité" ?» (p. 247).

\section{BIBLIOGRAPHIE}

BADCOCK B. (1989), « An Australian view of the rent gap hypothesis », Annals of the Association of American Geographers, vol.79, pp. 125-45.

BEAUREGARD R.A. (1984), « Structure, agency, and urban redevelopment », pp. 51-72 in M.P. SMITH (ed.) Cities in transformation, vol. 26, Urban Affairs Annual Reviews, London, Sage.

BEAUREGARD R.A. (1986), « The chaos and complexity of gentrification », pp. 35-55 in SMITH N. \& WILLIAMS P.(eds) Gentrification of the city, London, Allen and Unwin.

BERRY B.J.L. (1985), « Islands of renewal in seas of decay » in PEDERSON P. (ed.), The new urban reality, Washington, D.C, The Brookings Institute. 
BODDY M. (1976), « The structure of mortgage finance : building societies and the British social transformation », Transactions of the Institute of Bristish Geographers, N.S.1, pp. 58-71.

CLARK E. (1988), « The rent gap and transformation of the built environment : case studies in Malmo, 1860-1985 », Geografiska Annaler, vol.70.B, pp. 241-54.

Cibriwsky R.A., D.Ley D. \& Western J. (1986), « The politics and social construction of revitalized neighborhoods, Society Hill, Philadelphia and False Creek, Vancouver », pp. 92-120 in Smith N. \& Williams P. (eds) Gentrification of the City, London, Allen and Unwin.

Dingemans D.J.(1978), « Redlining and mortgage lending in Sacramento, California », Annals of the Association of American Geographers, vol.69, pp. 225-39.

Duncan J. \& Ley D. (1982), « Structural marxism and human geography : a critical assessment », Annals of the Association of American Geographers, vol.72, pp. 30-59.

Goetze R. (1979), Understanding neighbourhood change, Coalbridge, Mass., Ballinger.

GREGORY D.(1981), « Human agency and human geography », Transactions of the Institute of British Geographers, N.S.6, pp. 1-18.

HAMNETT C. (1984), « Gentrification and residential location theory : a review and assesment » pp. 283-319 in Herbert D.T. \& Johnston R.J. (eds), Geography and the urban environment. Progress in research and applications, vol.6, London, John Wiley.

Hamnett C. \& Randolph W. (1986), « Landlord disinvestment and housing market transformation : the flat break-up market in London ", pp. 121-52 in Williams P. \& Smith N. (eds.), Gentrification of the city, London, George Allen and Unwin.

Hamnett C. \& Randolph W. (1988), Cities, housing and profits, London, Hutchinson.

HARTMAN C. (1979), « Comment on neighbourhood revitalization and displacement : a review of the evidence ", Journal of the American Planning Association,.45, pp. 488-94.

HARVEY D. (1974), «Class monopoly rent finance capital and the urban revolution », Regional Studies, vol.8, pp. 239-55.

HARVEY D. (1978), «The urban process under capitalism : a framework for analysis », International Journal of Urban and Regional Research, vol. 2, pp. 101-31

HOYT H. (1939), The structure and growth of residential neighbourhoods in American cities, Washington D.C., Federal Housing Administration.

KING R.J. (1989c), « Capital switching and the role of ground rent : 3. Switching between circuits, switching between submarkets and social change ", Environment and Planning A, vol.21, pp. 853-880.

Legates R.T. \& Hartman C. (1986), « The anatomy of displacements in the United States », pp. 178-203 in Smith N. \& Williams P. (eds), Gentrification of the city, London, Allen and Unwin, London.

LEY D. (1978), «Inner city resurgence units societal context », mimeo, paper presented to the A.A.G. Annual Conference, New Orleans.

LEY D. (1980), « Liberal ideology and post industrial city ", Annals of the Association of American Geographers, vol.70, pp. 238-58.

LEY D.(1981), « Inner city revitalization in Canada : a Vancouver case study », Canadian Geographer, vol.25, pp. 124-48. 
LEY D. (1986), « Alternative explanations for inner city gentrification : A Canadian assessment », Annals of the Association of American Geographers, vol.76, pp. 521-35.

LEY D. (1987), « Reply : the rent gap revisited », Annals of the Association of American Geographers, vol.77, pp. 465-468.

MARCUSE P. (1986), « Abandonment, gentrification and displacement : the linkages in New York City ", pp. 153-177 in Smith N. and Williams P. (eds), Gentrification of the city, London, Allen and Unwin.

MOORE P.W. (1982), « Gentrification and the residential geography of the New Class », mimeo, Scarborough College, University of Toronto.

MULLINS P. (1982), « The "middle-class" and the inner city », Journal of Australian Political Economy, vol.11, pp. 44-58.

MUNT I. (1987), « Economic restructuring, culture and gentrification : a case study of Battersea, London », Environment and Planning A, vol.19, pp. 1175-1197.

ROSE D. (1984), « Rethinking gentrification : beyond the uneven development of marxist urban theory », Society and Space, vol.2, pp. 47-74.

ROSE D.(1989), «A feminist perspective on employment restructuring and gentrification : the case of Montreal », pp. 118-138 in Wolch. J. \& Dear M. (eds), The power of geography : how territory shapes social life, London, Unwin Hyman.

SCHAFFER R. \& SMITH N. (1986), « The gentrification of Harlem ? ", Annals of the Association of American Geographers, vol.76, pp. 347-365.

SMITH A. (1989), « Gentrification and the spatial constitution of the State : the restructuring of London's Docklands ", Antipode, vol.21, pp. 232-60.

SMITH N. (1979), « Toward a theory of gentrification : a back to the city movement by capital, not people ", Journal of the American Planning Association, vol.45, pp. 538-548.

SMITH N. (1982), « Gentrification and uneven development », Economic Geography, vol.58, pp. 139-155.

SMITH N. (1986), « Gentrification, the frontier, and the restructuring of urban space », pp. 15-35 in Smith N. \& Williams P.(eds), Gentrification of the city, London, Allen and Unwin.

SMITH N.(1987a), «Of yuppies and housing : gentrification, social restructuring and the urban dream », Society and Space, vol.5, pp. 151-172.

SMITH N.(1987b), «Gentrification and the rent gap », Annals of the American Association of Geographers, vol.77, pp. 462-478.

SMITH N.(1987c), « Dangers of the empirical turn », Antipode, vol.19, pp. 59-68.

SMITH N. \& Williams P. (eds) (1986), Gentrification of the city, London, Allen and Unwin.

Sternlieb G. \& Hughes J.W. (1983), « The uncertain future of the central city », Urban Affairs Quarterly, vol.18, pp. 455-472.

SUMKA H.(1979), « Neighborhood revitalization and displacement : a review of the evidence ", Journal of the American Planning Association, vol.45, pp. 480-487.

Walker R. \& Greenberg D. (1982), « Post-industrialism and political reform in the city : a critique », Antipode, vol.14, pp. 17-32. 
WILLIAMS P.( 1978), « Building societies and the inner city », Transactions of the Institute of British Geographers, vol.3, pp. 23-34.

WILLIAMS P. (1984), « Economic processes and urban change : an analysis of contemporary patterns of residential restructuring », Australian Geographical Studies, vol.22, pp. 39-57.

Wolfe J., DroverG. and Skelton I. (1980), « Inner city real estate activity in Montreal : institutional characteristics of decline », Canadian Geographer, vol.24, pp. 348-367.

ZUKIN S. (1982), Loft living: culture and capital in urban change, Baltimore, John Hopkins University Press.

ZUKIN S. (1987), « Gentrification : culture and capital in the urban core », American Sociological Review, vol.13, pp. 129-147.

\section{RÉSUMÉS}

Dans ce texte sont présentées les principales théories sur la gentrification proposées au cours de la dernière décennie, ainsi que le débat qui s'est développé autour d'elles. La gentrification a fait l'objet de nombreuses polémiques, parce qu'il s'agit d'un des champs de bataille théoriques les plus importants en géographie humaine, qui met en lumière les oppositions entre structure et action, production et consommation, capital et culture, offre et demande. Mais chacune des deux grandes explications qui ont été avancées pour rendre compte du processus de gentrification est une explication partielle, nécessaire mais non suffisante. Une explication complète de la gentrification doit à la fois tenir compte de la production des quartiers dévalorisés et de logements dégradés, et de la production de gentrifieurs et de leurs modes spécifiques de consommation et de reproduction.

\section{The blind men and the elephants : the explanation of gentrification}

This paper critically reviews the major theories of gentrification which have emerged over the last 10 years and the debate which has surrounded them. It argues the reason why the gentrification debate has attracted so much interest, and has been so hard fought, is that is one of key theoretical battlegrounds of contemporary human geography which highlights the arguments between structure and agency, production and consumption, capital and culture, and supply and demand. It also argues that each of the two major explanations which have been advanced to account for gentrifrication (the rent gap and the production of gentrifiers) is a partial explanation necessary but not sufficient. Finally, it argues that an integrated explanation for gentrification must involve both explanation of the production of devalued areas and housing and the production of gentrifiers and their specific consumption and reproduction patterns.

\section{INDEX}

Mots-clés : Espace, gentrification, logement, division sociale de l'espace, changement social Keywords : social change, housing, residential change 


\section{AUTEUR}

\section{CHRIS HAMNETT}

Chris HAMNETT est professeur de géographie à King's College (Londres). C'est un spécialiste de la division sociale de l'espace dans le grand Londres, et des interrelations entre marchés du logement et du travail dans les grandes métropoles. Il est l'auteur de nombreux articles et de plusieurs ouvrages sur Londres, sur l'évolution des structures du marché de logements, mais aussi sur les processus de polarisation sociale dans les grandes métropoles. 